\title{
UNE NOUVELLE DIASTASE DANS LE LAIT : LA FIBRINOGÉNASE
}

\author{
par
}

\section{LASCAR BURUIANA}

Laboratoire de Chimie biologique. Faculté Vétérinaire de Bucarest.

Hamburger et Moro, ainsi que Bernheim et Karrer, ont. observé, déjà depuis 1902, que le lait de femme a la propriété de coaguler le liquide d'hydrocèle du nouveau-né.

Plus tard, A. Sole (1) a constaté que ce lait abrège le temps de coagulation du sang veineux. D'après cet auteur, les laits de vache, de chèvre, de cobaye et de jument, ne possèdent pas cette propriété. Il constate, en outre, que l'agent responsable de cette action, considéré, d'après lui, comme différent de la thrombine, est absorbé par les globules gras du lait. Plus récemment, W. KRAszewski et L. LINDENFELD (2), reprenant l'étude de cette question, arrivent à la. conclusion que l'action coagulante du lait est due à la présence de la cytosime qui accélère la formation de la thrombine. Ayant trouvé que le lait de femme ne coagule pas le sang oxalaté ou citraté ainsi que les solutions de fibrinogène, ils nient l'existence de la thrombine. ou de la prothrombine dans ce liquide. Tout en confirmant les expériences de Kraszewski et Lindenfeld, F. Bellemli (3) affirme que la salive et l'urine possèdent la même propriété que le lait vis-à-vis du sang et que cette action n'est pas empêchée par l'ébullition de ces liquides biologiques. Enfin, M. JАСоBY et S. ADLER (4) trouvent que le lait de vache n'exerce aucune action coagulante sur le sang. Ils supposent que dans les cas où cette action est manifeste, elle serait due aux ions calcium.

$$
\text { *** }
$$

Afin d'éclairer le mécanisme du phénomène signalé et par là même d'interpréter les conclusions assez souvent contradictoires des auteurs qui s'en sont occupé jusqu'ici, nous avons entrepris une série d'expériences. Ce sont les résultats de ces expériences. que nous voulons exposer dans cette note.

Sur des solutions de fibrinogène à $0,6 \%$ (préparé suivant la. méthode de Gabriel Bertrand (5)) qui coagulent à $48^{\circ}$, nous avons. essayé l'action du lait de différentes espèces. Les essais ont été

(1) Klinische Wschr., 1935, 14, 1354-1358.

(2) Klinische Wschr., 1935, 24, 363 et Presse Médicale, 1935, 68, 1332-1337.

(3) Riforma Medica, 1937, 32, 1138.

(4) Enzymologia, 1937, 1, 373.

(5) Guide pour les manipulations de Chimie biologique, 1919. 
toujours faits à la température du laboratoire $\left(18-20^{\circ}\right)$ et dans des conditions variables quant à la concentration en ions hydrogène du milieu. Il ressort de ces expériences que l'influence de la concentration en ions hydrogène est le facteur fondamental dont dépend l'activité coagulante du lait et que son optimum se trouve à $p \mathrm{H}=7$. Faute d'avoir négligé ce facteur, on est arrivé à des conclusions comme celle-ci : le lait de femme possède l'activité la plus intense, tandis que ceux de lapin et de chèvre en sont dépourvus. En ajustant le $p \mathbf{H}$ à l'optimum, c'est-à-dire à $p \mathbf{H}=7$, les résultats sont tout à fait différents.

En voici quelques exemples:

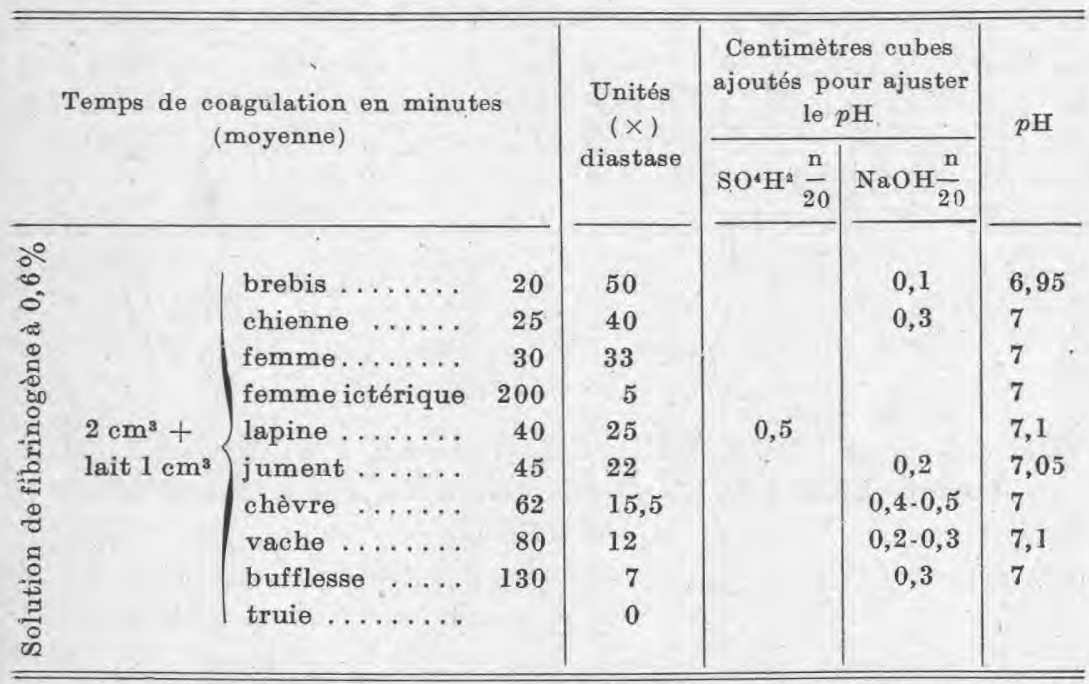

Comme on le voit, le lait de truie est dépourvu de propriété coagulante pour le sang. $\mathrm{Y}$ aurait-il une corrélation entre cette absence et la richesse en vitamine $\mathrm{R}$ (antihémorragique) de la graisse du foie chez cette espèce?

$$
* * *
$$

Du lait de brebis dont l'activité coagulante vis-à-vis de la solution de fibrinogène s'est montrée la plus intense, nous avons réussi à préparer un extrait doué d'une activité de beaucoup plus intense que celle du lait. Dans ce but, nous avons mis à profit la propriété, de la membrane haptogène des globules gras du lait, d'absorber certaines diastases. La technique suivie a été celle que nous avons employée autrefois pour isoler la réductase de ScHaR-

( $\times$ ) L'inverse du temps de coagulation multiplié par 1.000. 
DINGER (1). Vu le fait que l'extrait isolé de cette manière est thermolabile et en même temps très sensible aux variations de concentration en ions hydrogène, nous sommes persuadé qu'il s'agit dans. ce cas d'une vraie diastase. Pour cette diastase nous proposons, pour nous conformer à l'usage établi, le nom de fibrinogénase.

$$
*^{*} *
$$

En dehors de cette diastase, nous avons été conduit à mettre en évidence encore un facteur existant dans le lait et possédant la propriété de coaguler les solutions de fibrinogène. Il s'agit cette fois d'une substance se trouvant dans la fraction non saponifiable de l'extrait éthéré de la matière grasse du lait.

L'action de cette substance sur les solutions de fibrinogène nese manifeste pas par une coagulation massive comme cela se passe avec la fibrinogénase, mais par un phénomène de précipitation du fibrinogène. Comme ce dernier facteur est plus résistant à la chaleur, il se pourrait qu'il ne soit rien d'autre que la vitamine $K$.

Cette supposition paraît être corroborée par l'expérience avec le lait de femme ictérique, pour la raison que, dans l'ictère l'absorption intestinale des graisses étant entravée, il se pourrait que la synthèse de la vitamine $\mathrm{R}$ soit, elle-même, troublée sinon supprimée.

\section{SUR LA RUPTURE DE L'ÉQUILIBRE PHOSPHATIQUE DANS LES LAITS MICROBIENS FORTEMENT CHAUFFÉS}

\section{CAS DES LAITS STÉRILISÉS (2) \\ par}

\section{G. GUITTONNEAU}

Professeur à l'Institut agronomique,

Directeur

\section{Mile M. BEJAMBES}

Chef de travaux

du Laboratoire national des Industries laitières.

\section{Introduction.}

Nous avons précédemment montré [1] que, chauffés dans du: lait cru de vache, un certain nombre de microorganismes, les ferments lactiques en particulier, retenaient par adsorption les phosphates calciques libérés des complexes phosphocaséinatiques du lait. à la faveur des traitements thermiques.

Le revêtement de phosphates ainsi fixé à la surface des corps microbiens est tel qu'il peut les protéger contre l'action des colorants.

(1) Buruiana. Recherches potentiométriques sur l'enzyme de Schardinger. Thèse, Bucarest, 1935.

(2) Publié également dans les Annales de Technologie agricole, t. III, 1940. 